\title{
Combining structured and unstructured data in EMRs to create clinically-defined EMR-derived cohorts
}

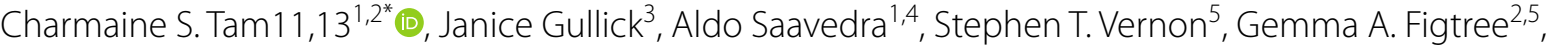 \\ Clara K. Chow ${ }^{6,7}$, Michelle Cretikos ${ }^{9}$, Richard W. Morris ${ }^{1,2}$, Maged William ${ }^{10}$, Jonathan Morris ${ }^{2,8}$ \\ and David Brieger ${ }^{12}$
}

\begin{abstract}
Background: There have been few studies describing how production EMR systems can be systematically queried to identify clinically-defined populations and limited studies utilising free-text in this process. The aim of this study is to provide a generalisable methodology for constructing clinically-defined EMR-derived patient cohorts using structured and unstructured data in EMRs.

Methods: Patients with possible acute coronary syndrome (ACS) were used as an exemplar. Cardiologists defined clinical criteria for patients presenting with possible ACS. These were mapped to data tables within the production EMR system creating seven inclusion criteria comprised of structured data fields (orders and investigations, procedures, scanned electrocardiogram (ECG) images, and diagnostic codes) and unstructured clinical documentation. Data were extracted from two local health districts (LHD) in Sydney, Australia. Outcome measures included examination of the relative contribution of individual inclusion criteria to the identification of eligible encounters, comparisons between inclusion criterion and evaluation of consistency of data extracts across years and LHDs.

Results: Among 802,742 encounters in a 5 year dataset (1/1/13-30/12/17), the presence of an ECG image (54.8\% of encounters) and symptoms and keywords in clinical documentation (41.4-64.0\%) were used most often to identify presentations of possible ACS. Orders and investigations (27.3\%) and procedures (1.4\%), were less often present for identified presentations. Relevant ICD-10/SNOMED CT codes were present for 3.7\% of identified encounters. Similar trends were seen when the two LHDs were examined separately, and across years.

Conclusions: Clinically-defined EMR-derived cohorts combining structured and unstructured data during cohort identification is a necessary prerequisite for critical validation work required for development of real-time clinical decision support and learning health systems.
\end{abstract}

Keywords: Electronic medical record, Cohort identification, Electronic phenotype, Acute coronary syndrome

*Correspondence: charmaine.tam@sydney.edu.au

${ }^{1}$ Centre for Translational Data Science, The University of Sydney, Sydney,

Australia

Full list of author information is available at the end of the article

\section{Background}

The widespread adoption of electronic medical records (EMR) offers unprecedented opportunities to rapidly ascertain and examine clinical data at large-scale and low cost; such information is essential for applications such as audit and feedback, near real-time clinical decision support as well as supporting research objectives through cohort studies, registries and large-scale 
pragmatic clinical trials [1-3]. Administrative coding systems such as International Classification of Diseases and Related Health Problems (ICD)-10 provide a translation of healthcare diagnoses, procedures, medical services, and medical equipment into universal codes [4], however do not provide a granular view of a patient's presentation, severity of disease and clinical sequence during an episode of care [4-6] and have variable accuracy [7]. As such, improved computational methods which maximise the depth and accuracy of information extracted from production-level EMR systems are essential to fulfil the promise of real-time clinical decision support which rely on a reliable knowledge base to guide clinical decision making within a learning health system [8].

The development of robust methodologies that enable identification of clinical-defined cohorts from the overall patient population captured in production EMR systems (e.g. Cerner, Epic) are a critical first step [9]. The most straightforward approach is to use clearly defined events or procedure codes (e.g. type of surgery, cancer diagnosis) associated with the hospitalisation to identify cohorts $[10,11]$. However, diagnostic codes alone are insufficient for identifying clinical EMR-derived cohorts due to strict rules adhered to for coding, underreporting and the complexity of diseases being assigned a single code [12]. The cohort identification process becomes even more challenging when diseases and conditions have heterogeneous aetiology and a spectrum of severity [13-15]. Approaches to data extraction from production EMR systems also have to consider whether there is ready access to production-level EMR environments (e.g. live systems, back-ups, copies of the production EMR etc.) which may limit the granularity and timeliness of information that is available for cohort identification as well as the opportunity for iteration during the cohort identification process.

There have been few reports describing how production EMR systems can be systematically queried to identify and reliably extract information from a clinicallydefined cohort of interest and limited studies leveraging the $>70 \%$ of the EMR that is captured in free-text during this process [16-20]. Furthermore, previous studies have used structured data fields (e.g. ICD-10 code) to identify the population for data extraction which may lead to eligible patients being missed during data extraction if an ICD-10 code was absent or mis-coded [21, 22] or cohort identification has occurred after the data has been extracted from the production EMR system, which would also result in missed eligible patients [12, 23]. Mis-coding would lead to measurement error and missing data would contribute to selection bias and counteract the statistical power available from leveraging data housed in EMRs.

The aim of this study was to develop a generalisable methodology used within production EMR systems for creating high-fidelity clinically-derived EMR cohorts for complex diseases/conditions which cannot exclusively use diagnostic or procedure codes to identify a cohort of interest. Patients with possible acute coronary syndrome (ACS) were selected as an exemplar given its heterogeneous aetiology and spectrum of severity during presentation. This approach can be applied to other complex diseases/conditions including but not limited to mental illness, asthma, rheumatoid arthritis, chronic kidney disease $[13-15,19]$.

\section{Methods}

\section{Source population}

The eight metropolitan and seven rural/regional Local Health Districts (LHD) in New South Wales (NSW), Australia are responsible for managing public hospitals and health institutions and for providing health services within a geographical area. The source population presented to health care facilities in Northern Sydney LHD and Central Coast LHD, two of the eight metropolitan LHDs in NSW. Within Northern Sydney LHD and Central Coast LHD, there were eight publicly-funded hospitals that admitted patients with possible ACS. This included two tertiary hospitals with 24-h percutaneous coronary intervention capability and six referral hospitals; all of which used Cerner Millennium information systems. These two LHDs had a combined estimated resident population of 1.26 million people [24]. In this protocol paper, we followed the guidelines developed in the RECORD statement for reporting studies conducted using observational, routinely-collected health data [25].

\section{Study inclusion criteria and rationale}

The methodology for cohort identification is summarised in Fig. 1. A multi-disciplinary team consisted of 5 clinicians (cardiologists, population health physicians, nurses) and 5 electronic data experts (data engineers, business analysts, data analysts, analytics translator). The multidisciplinary team varied in experience from early career through to professor level with career stages equally represented (Step 1, Fig. 1). In a series of initial meetings, the cardiologists defined clinical criteria to identify patients presenting with possible ACS (Step 2, Fig. 1). The data experts then mapped the clinical criteria to discrete data tables within the Cerner Millennium EMR system to locate the data elements required for identification of patients using these clinical criteria (Step 3, Fig. 1). This mapping process was discussed and agreed upon at iterative meetings over a 3-month period with the multi-disciplinary team (Step 4, Fig. 1). This approach of using a multi-disciplinary team to define clinical criteria which can then be mapped to discrete data tables in the EMR can be applied to any condition/ 


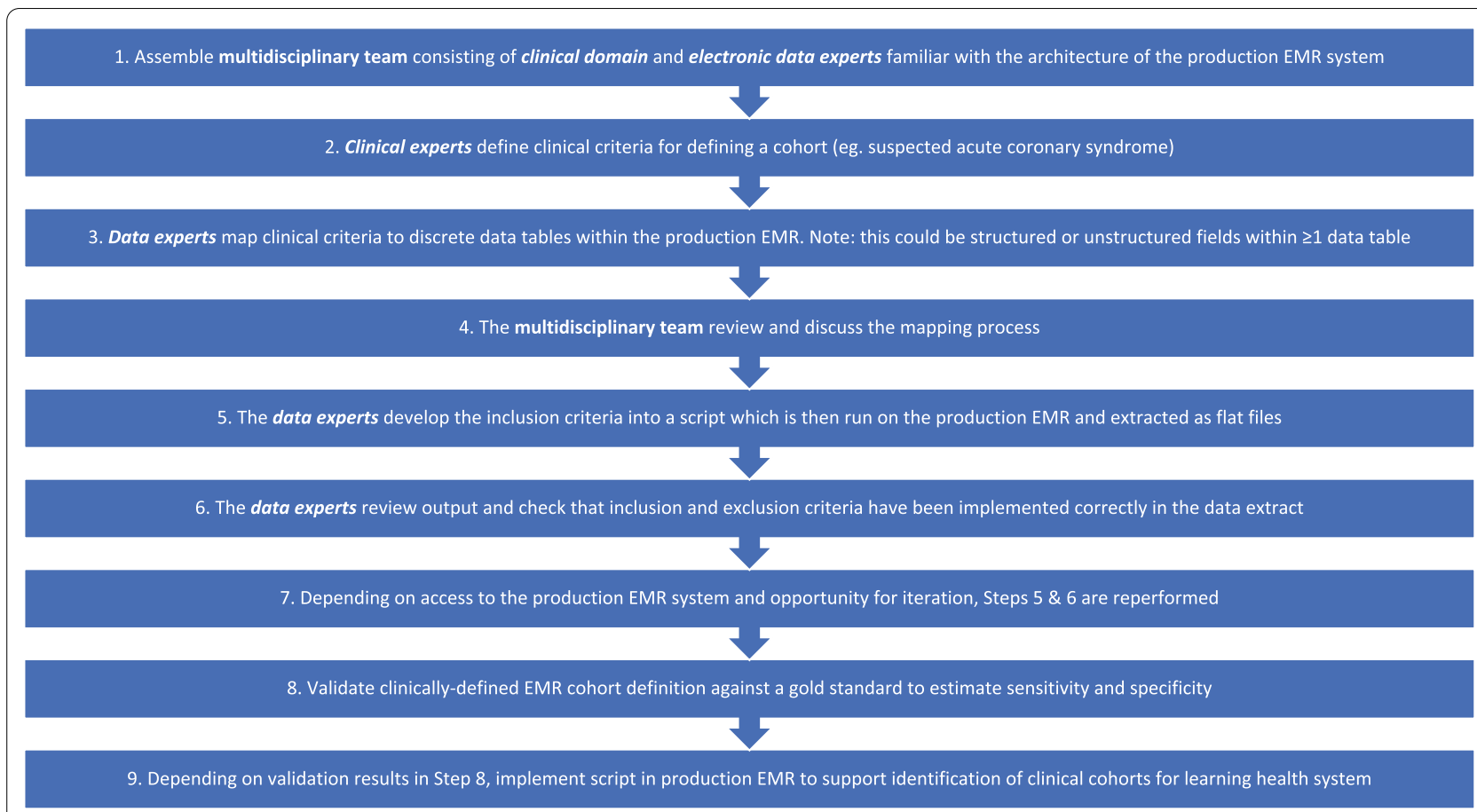

Fig. 1 General methodology for creating clinically-defined cohorts using structured and unstructured data in production EMR systems

disease of interest. During this process, we considered the previously described phenotype algorithm model workflow model for portable algorithms [26], against existing technological constraints for extracting data from Cerner EMR systems within our health jurisdiction (Steps 5-7, Fig. 1). The next steps would involve validation of a clinically-defined EMR cohort against a gold standard to estimate sensitivity and specificity for a diagnosis. Depending on whether the sensitivity and specificity results were deemed clinically acceptable, the script would then be implemented in a production EMR environment to support identification of clinical cohort for learning health systems (Steps 8-9, Fig. 1). The methods of such validation studies and implementation into production EMR systems are beyond the scope of the current study but are a critical step prior to implementation within learning health systems.

For the current study, seven inclusion criteria to identify possible ACS were developed to identify the cohort of interest (Table 1). If any of the seven inclusion criteria

\section{Table 1 Study inclusion criteria}

\begin{tabular}{|c|c|}
\hline $\begin{array}{l}\text { Inclusion } \\
\text { criteria }\end{array}$ & Definition \\
\hline 1 & $\begin{array}{l}\text { The "Reason for Visit" (free text field) for the presentation contained any of the ACS-related symptoms or keywords described in Additional } \\
\text { file 1: Information Part } 1\end{array}$ \\
\hline 2 & $\begin{array}{l}\text { The patient was placed on a cardiac pathway care plan OR the information collected during emergency department triage (free-text), } \\
\text { separate to the Presenting Information field above, contained any of the list of ACS-related symptoms or keywords (Additional file 1: } \\
\text { Information Part 1) }\end{array}$ \\
\hline 3 & $\begin{array}{l}\text { Orders were placed in the EMR for a troponin test OR a } 12 \text { lead ECG OR for any of the following investigations: coronary angiogram, exercise } \\
\text { stress test, stress echocardiogram, sestamibi scan, CT coronary angiogram, CT pulmonary angiogram }\end{array}$ \\
\hline 4 & The patient's EMR contained a "Cardiac Monitoring" form, meaning that the patient had been placed on a cardiac monitoring pathway \\
\hline 5 & $\begin{array}{l}\text { The patient had a result recorded in the EMR from a sestamibi scan, CT coronary angiogram, CT aortic angiogram or CT pulmonary angio- } \\
\text { gram }\end{array}$ \\
\hline 6 & 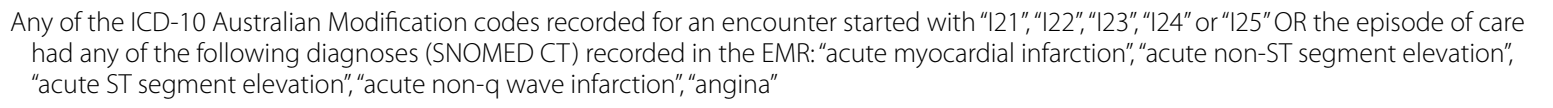 \\
\hline 7 & The encounter contained a scanned 12-lead ECG image \\
\hline
\end{tabular}


were met for an encounter during the study period, the encounter (termed an "eligible" encounter) was deemed eligible for inclusion into the study population. An encounter was defined as an electronically recorded interaction between a patient and healthcare provider, characterised by a unique identifier and admission time. Once an encounter met any of the seven inclusion criteria, all clinical, biochemical and demographic information contained within the EMR were extracted. This also included information from "reference" encounters for the individual patient dating back to 2002 when the EMR system was first implemented in the health district.

For criteria (1) and (2), an initial list of ACS-related symptoms, keywords and abbreviations, for patients presenting with possible ACS was developed in consultation with the clinical reference group. The list was extended by manual review of $\sim 50$ Emergency Department triage forms in patients with an ICD-10 code for STEMI (I21.0, I21.1, I21.2, I21.3) or NSTEMI (I21.4). Next, we examined the frequency of each of the search terms for relevant data fields within a subset of ED triage forms obtained from a 3-month test extract ( $\mathrm{n} \sim 30,000$ encounters between $1 / 4 / 17$ to $30 / 6 / 17$ ). Given the unstructured nature of free-text, we also identified and included misspellings and abbreviations for each of the terms explicitly due to the text processing limitations of Cerner Command Language (CCL) used to extract the data; regular expressions were not able to be used in CCL. If the keywords were present at least 20 times (chosen as an arbitrary cut-off) in the subset of 30,000 ED Triage forms, they were then included in the final list of search terms of ACS-related symptoms and keywords.

\section{EMR data extraction}

Data extraction was performed for a single continuous five-year time period between 1/1/13 and 31/12/17 . Encounters were extracted through the execution of a bespoke CCL script which contained seven functions (representing the seven inclusion criteria) which were designed to identify eligible encounters. Data extraction was performed by an external party (MKM Health, Chatswood, NSW) and in line with the HREC approval, the study investigators had no direct access to the EMR information systems or contained within the EMR. The external party wrote the CCL script which was then run on the production EMR system. Then, flat data tables were created, de-identified and extracted from the production EMR for the research team for analyses (Fig. 2). Approaches for ensuring data quality and the operational framework of the study (data management, security and governance) are described in Additional file 1: Information, Part 2.

\section{Outcome measures}

EMR data extracts were processed and analysed using $R$ (Version 4.0.0). Outcome measures included 1) examination of the relative contribution of individual inclusion criteria to the identification of eligible encounters, 2) associations between the use of diagnostic codes alone (ICD-10/SNOMED Clinical Terms (CT)) and other inclusion criteria and 3) examination of consistency across LHDs and time. First, we developed a computational method to check the composition of inclusion criteria met by each encounter. Each encounter received a score between 0 and 7 indicating how many of the inclusion criteria were met. Any encounter with a score ${ }^{3} 1$ was deemed eligible and included in the index cohort. Encounters that did not meet any of the inclusion criteria were assigned a value of ' 0 ' and referred to as "reference" encounters (i.e. they are not eligible encounters). Next, we examined the proportions of encounters identified using diagnostic codes alone $v s$. other inclusion criteria, and vice-versa and a correlation matrix was used to calculate the associations between each of the inclusion criteria. Finally, we examined consistency in the composition of inclusion criteria across time and local health districts.

\section{Results}

Examination of the relative contribution of individual inclusion criteria to eligible encounters

The 5-year extract (1/1/13-31/12/17) consisted of 802,742 eligible and 5,418,466 reference encounters. Out of the 802,742 eligible encounters, scanned ECG images (54.8\% of encounters) and symptoms and possible ACSrelated keywords in clinical documentation (41.4-64.0\%) were used most often to identify presentations of possible ACS. Orders and investigations (27.3\%) and procedures (1.4\%), were less often present for identified presentations. Relevant ICD-10/SNOMED CT codes were present for $3.7 \%$ of identified encounters.

To further examine the composition of inclusion criteria in eligible encounters, UpSet plots [27] were used to represent the frequency of each inclusion criterion and the numbers of encounters that met each combination of inclusion criteria in 2017, the most recent data in our 5-year data extract. Figure 3 shows 185,414 eligible encounters in 2017 with similar findings as the total 5 year dataset. Individually, the presence of a scanned ECG image (72.0\%), the presence of keywords captured in the presenting information in the ED triage form $(60.0 \%)$ and the presence of keywords in the "Reason for visit" for the presentation (38.2\%) identified the majority of eligible encounters. Orders and investigations $(25.6 \%)$ and procedures $(1.2 \%)$, were less often present for eligible 


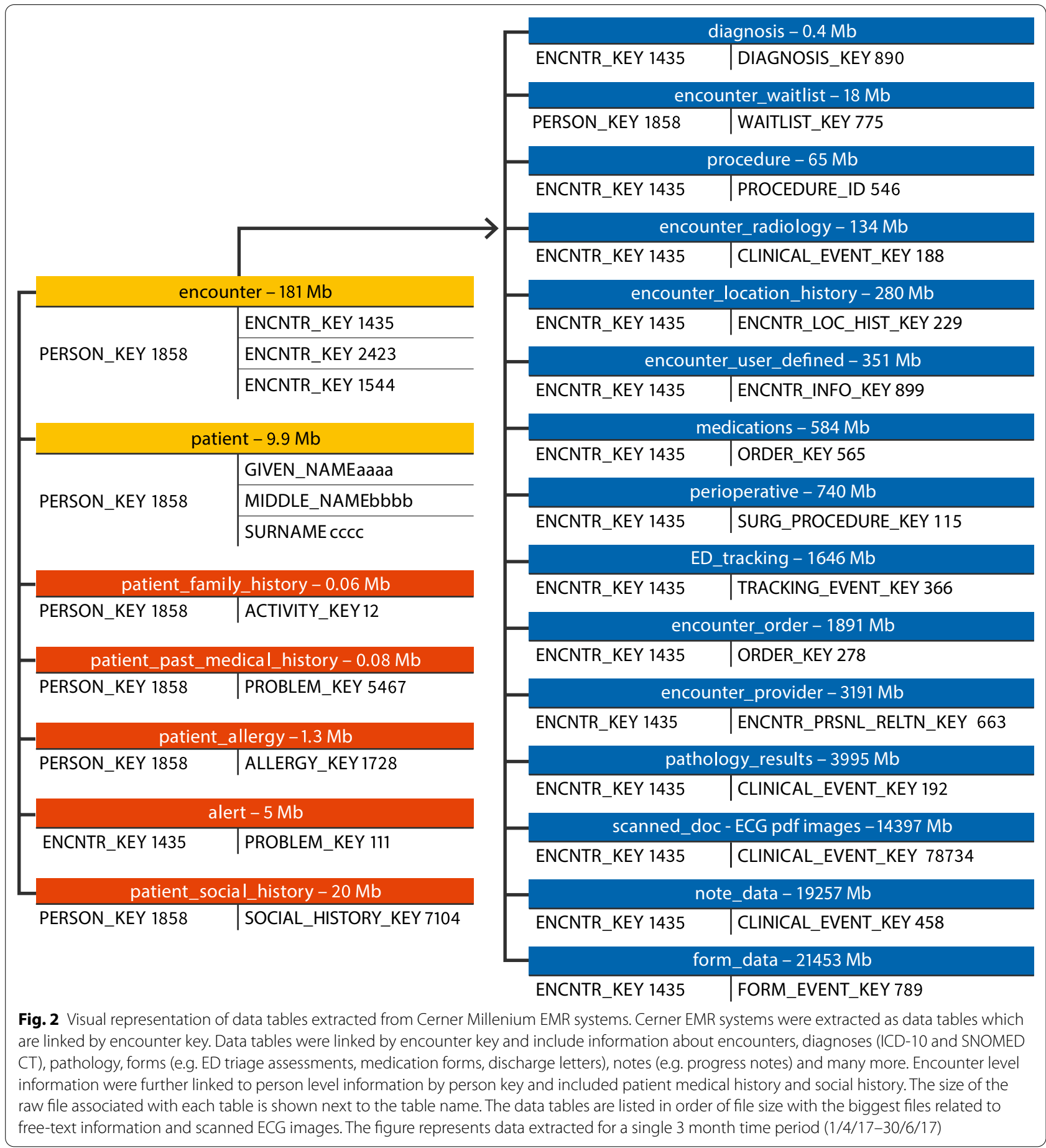

encounters. Only $3.1 \%$ of encounters had the presence of a relevant diagnostic code (ICD-10 or SNOMED CT) for ACS. The presence of a cardiac monitoring form was not an informative criterion for identifying eligible encounters.

We found 59 unique combinations of inclusion criteria met by encounters (Fig. 3). The most frequent combinations were encounters that only had the presence of a scanned ECG image (26\%; 47,514 of 185,414$)$, encounters that only had a keyword match in triage information $(18 \% ; 33,865$ of 185,414$)$ and encounters that had the presence of an ECG, keyword match in triage information and a relevant order $(12 \% ; 22,160$ of 185,414$)$. Similar trends were seen in UpSet plots 


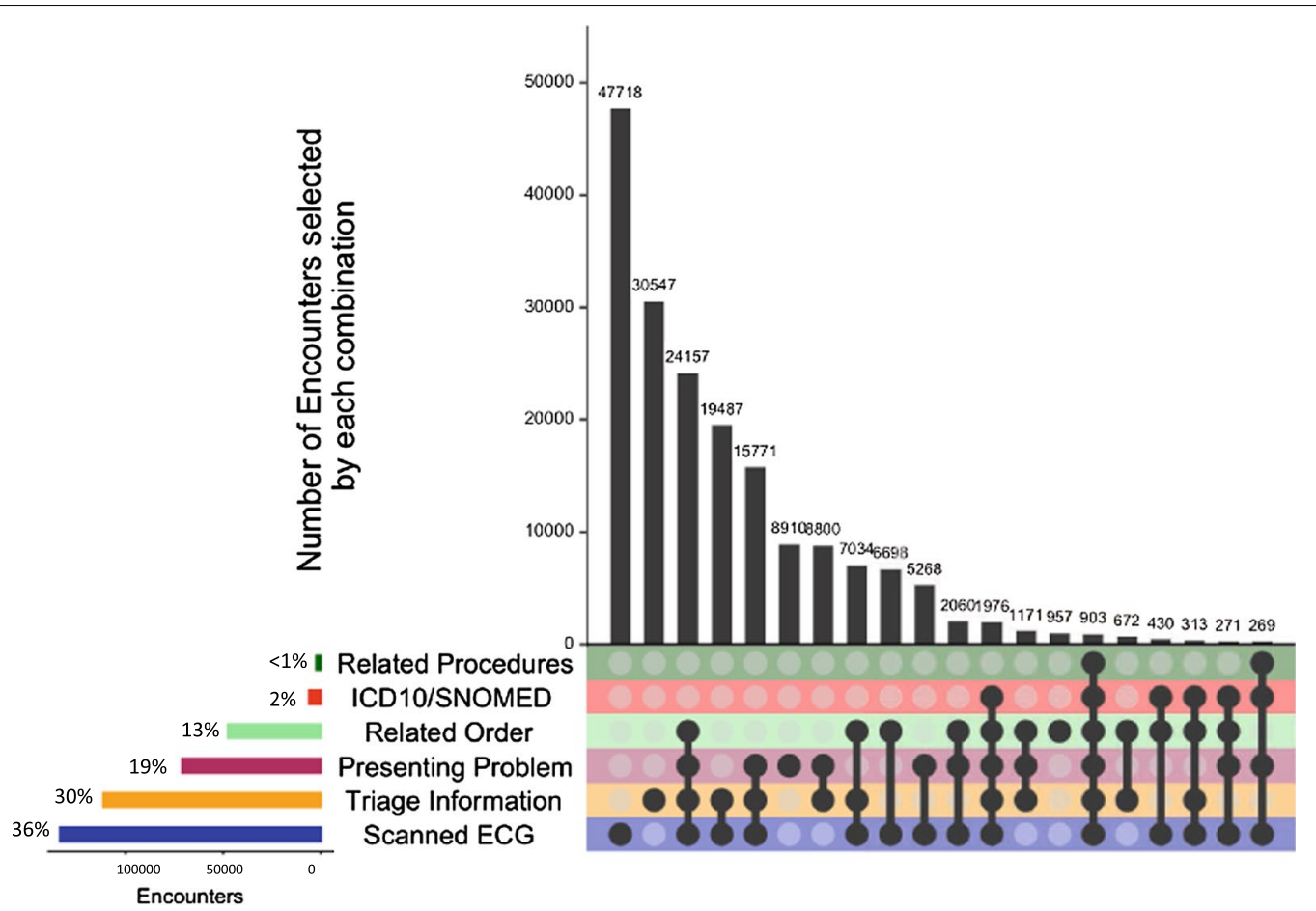

Fig. 3 UpSet plot showing the number of encounters meeting individual (bottom left hand side) and multiple inclusion criteria (right-hand side). This UpSet plot represents 317,719 eligible encounters from Cerner information systems in two local health districts that met at least one of the study inclusion criteria in 2017. Inclusion criteria were described in Table 1. The histogram on the bottom left-hand side represents the total number and percentage of encounters that met each inclusion criterion. The plot on the top right-hand side represents the number and percentage of encounters that met each unique combination of inclusion criteria, depicted by the black circle(s) and lines. For example, the most frequent combinations were encounters that only had the presence of a scanned ECG image, followed by encounters that only had a keyword match in triage information

performed for the other years (2013-6; Additional file 2: Figure S1).

\section{Comparisons between diagnostic codes and other inclusion criteria}

In the 5-year extract, we examined the proportion of encounters that contained a relevant diagnostic code within each cohort of encounters met by each inclusion criterion. Given the broad inclusion criteria developed for this study, diagnostic codes comprised a minor component of each individual inclusion criteria cohort (Fig. 4). Note that $0.1 \%(858 / 802.742)$ of eligible encounters were identified using diagnostic codes alone. A correlation matrix examining associations between each of the inclusion criteria found a strong correlation between diagnostic code and relevant procedures (Pearson's correlation coefficient $=0.9$ ), with small to moderate associations with the other inclusion criteria (Additional file 3: Figure S2).

\section{Consistency across local health districts and time}

To examine consistency of the methodology, we examined the frequency of each inclusion criterion for each year in our 5 year extract (Table 2). Individually, the presence of a scanned ECG image (15-36\%), the presence of keywords captured in the presenting information in the ED triage form (19-25\%) and the presence of keywords in the "Reason for visit" for the presentation (30-40\%) identified the majority of eligible encounters. Orders and investigations $(13-17 \%)$ and procedures $(<1 \%)$, were less often present for eligible encounters. $2-3 \%$ had the presence of a relevant diagnostic code (ICD-10 or SNOMED CT) for ACS. Similar trends were observed when the two LHDs were examined separately, with a diagnostic code being present in $2-3 \%$ of eligible encounters (Fig. 5).

\section{Discussion}

Our study demonstrates that informatics approaches combining structured EMR data, such as orders for pathology testing, investigations and diagnostic codes, 

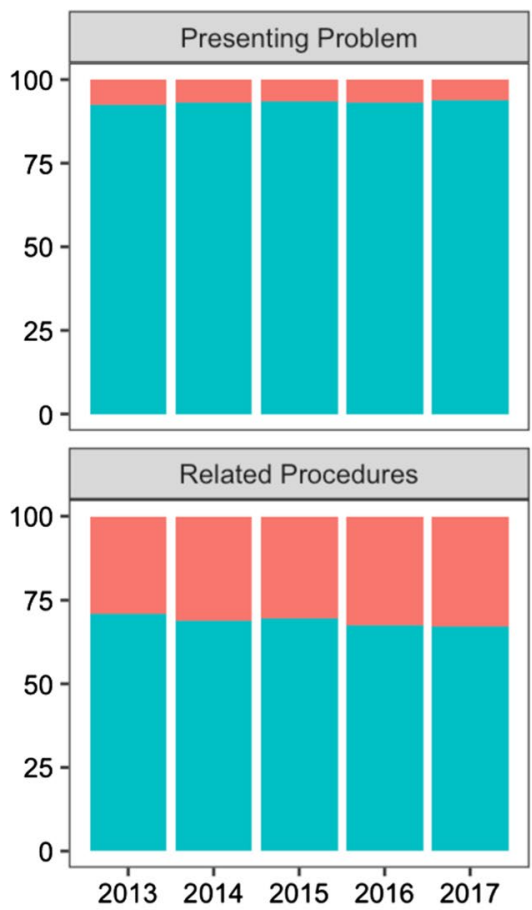
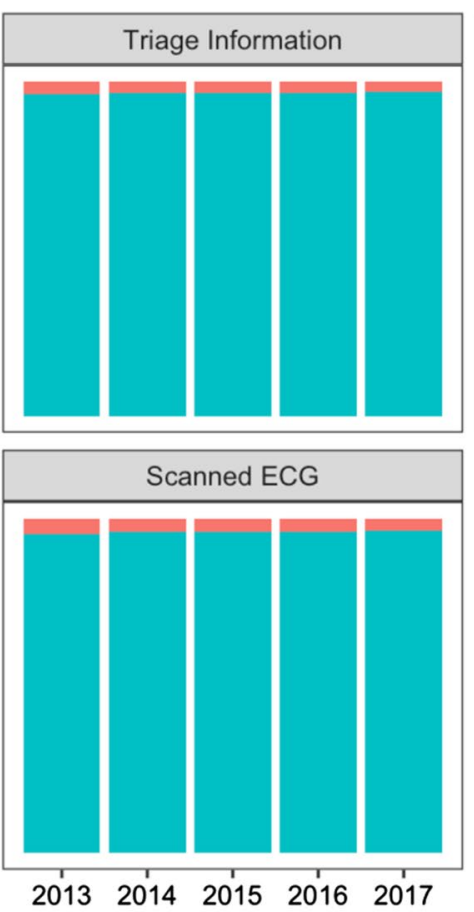

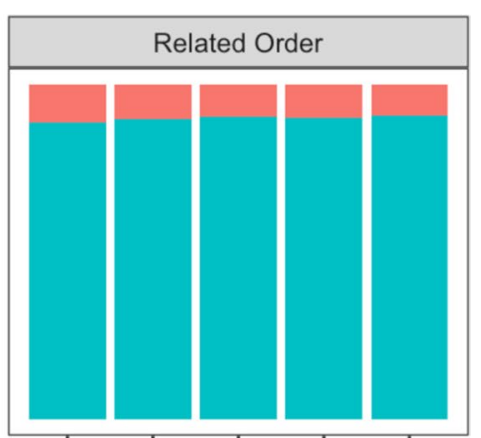

$\begin{array}{lllll}2013 & 2014 & 2015 & 2016 & 2017\end{array}$

with ICD10/SNOMED

without

Fig. 4 Percentage of eligible encounters with diagnostic codes (ICD-10/SNOMED CT) for each inclusion criteria, by year

Table 2 Percentage of encounters that met each inclusion criteria in the 5 year cohort

\begin{tabular}{lllllrr}
\hline Year & $\begin{array}{l}\text { Presenting } \\
\text { problem, } \mathbf{n}(\%)\end{array}$ & Triage information, $\mathbf{n}(\%)$ & Related order, $\mathbf{n}(\%)$ & $\begin{array}{l}\text { Related } \\
\text { procedures, } \\
\mathbf{n}(\%)\end{array}$ & $\begin{array}{l}\text { ICD-10/ } \\
\text { SNOMED CT, } \\
\mathbf{n}(\%)\end{array}$ & $\begin{array}{l}\text { Scanned ECG } \\
\text { image, } \mathbf{n}(\%)\end{array}$ \\
\hline $2013(n=135,511)$ & $61,529(45.4)$ & $98,045(72.3)$ & $41,629(30.7)$ & $2296(1.7)$ & $6240(4.6)$ & $36,902(27.2)$ \\
$2014(n=143,108)$ & $65,668(45.8)$ & $99,661(69.6)$ & $42,839(29.9)$ & $2259(1.6)$ & $5778(4.0)$ & $46,437(32.5)$ \\
$2015(n=163,008)$ & $66,428(40.8)$ & $100,048(61.4)$ & $42,734(26.2)$ & $2099(1.3)$ & $5613(3.4)$ & $101,363(62.2)$ \\
$2016(n=175,701)$ & $68,286(38.9)$ & $105,428(60.0)$ & $45,180(25.7)$ & $2353(1.3)$ & $5987(3.4)$ & $121,575(69.2)$ \\
$2017(n=185,414)$ & $70,878(38.2)$ & $111,275(60.0)$ & $47,566(25.7)$ & $2243(1.2)$ & $5768(3.1)$ & $133,429(72.0)$ \\
\hline
\end{tabular}

with symptom and keyword text mining in narrative freetext during the data extraction process in production EMR systems, can create high fidelity clinical-defined patient cohorts. This inclusive approach to EMR data extraction enables subsequent datasets for EMR-derived cohorts to be readily created and updated as new conditions/diseases emerge and clinical definitions are updated [28], as well as the extraction of clinically-relevant information enabling future validation studies of diagnostic and procedure codes, which are essential for real-time clinical decision support and secondary use of EMR data $[11,29,30]$. This is particularly significant for diagnoses which have a diverse range of presenting problems (e.g.
ACS, mental illness, sepsis) [31]. The use of diagnostic codes alone during this process would likely have led to relevant patients not being captured [32] as evidenced by the finding that $<1 \%$ of eligible encounters contained an ACS-related diagnostic code [29].

Our design approach for data extraction from the production EMR system balanced pragmatic and technological constraints against the broader study goal of creating a comprehensive EMR data platform that could be interrogated for a range of cardiovascular-related questions and other use cases in the future. For example, despite widespread use of regular expression and advances in natural language processing which would enable more 


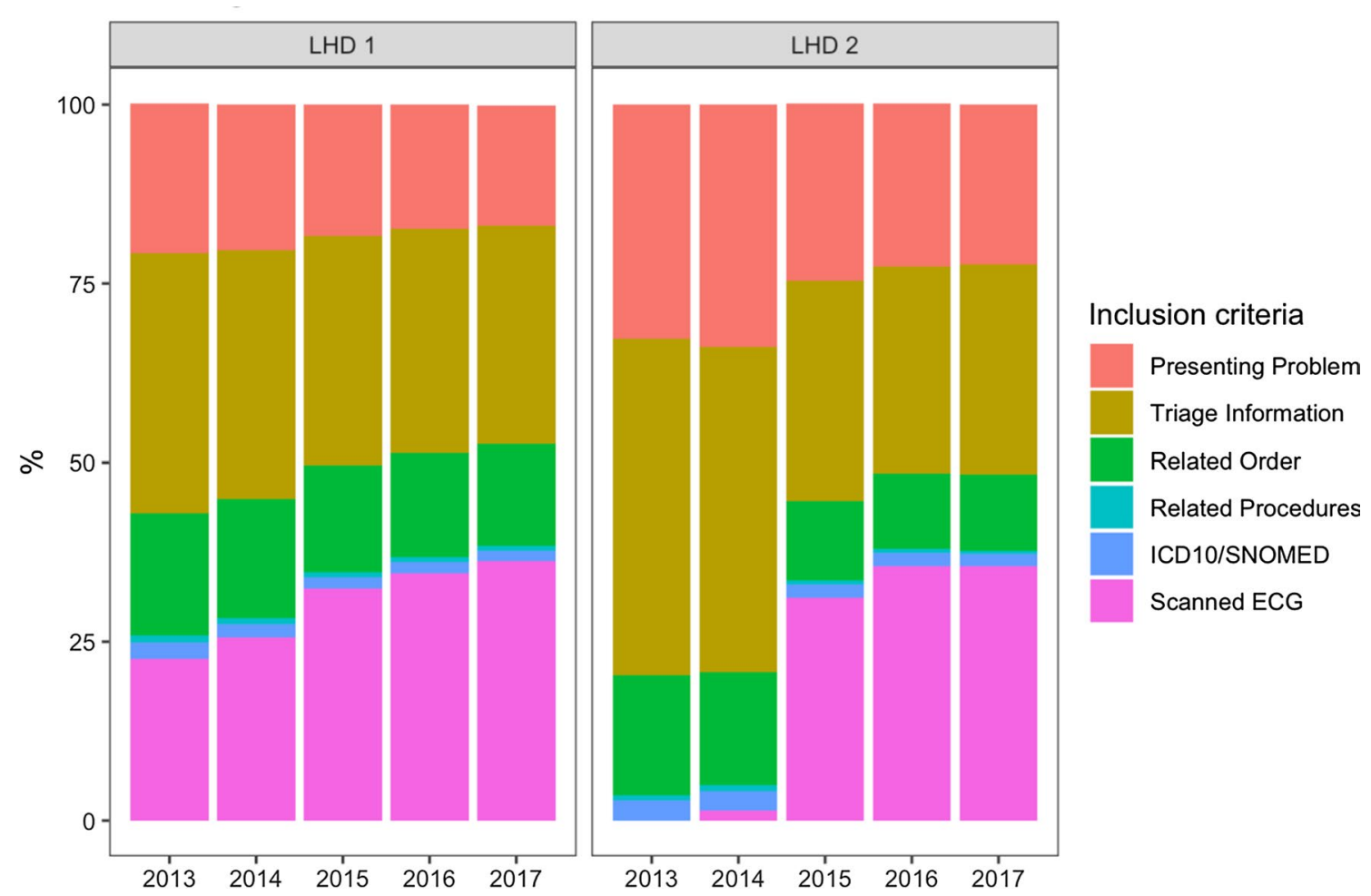

Fig. 5 Percentage of total encounters in each local health district that met each inclusion criteria in a 5 year dataset. LHD 1 refers to Northern Sydney Local Health District and LHD 2 refers to Central Coast Local Health District.

thorough identification of presenting symptoms in freetext $[33,34]$, this was not available in the Cerner EMR implementation at the LHDs in this study. As such, these techniques were not able to be used production EMR systems, although there is progress to integrate them in the future [35]. Machine learning methods require access to specialist libraries and often high performance computing and tend to only able to be implemented after data has been extracted from production EMR systems. This study also served as a proof-of-concept for demonstrating to the organisation that data housed within EMR systems could be readily extracted for clinical utility.

Our work builds on earlier studies combining structured and unstructured free-text EMR data for identifying cohorts after data has been extracted from production EMR systems, demonstrating that diagnostic codes alone are insufficient for disease case detection. Penz et al. found that ICD-9 and current procedure terminology (CPT) codes identified less than $11 \%$ of the cases in a study of detecting adverse events related to central venous catheters, while natural language processing methods achieved a sensitivity of 0.72 and specificity of 0.80 [36]. Similar findings have been observed for detecting colorectal cancer cases [12]. Our study findings demonstrate that similar approaches for identifying cohorts are required within production EMR systems themselves, which have also been demonstrated by large collaborative research networks (e.g. PCORnet, eMERGE, ODHSI) [37].

The generalisable methodology described in this study is essential for curation of high-fidelity clinical data from EMR systems enabling continuous, routine monitoring and reporting on the quality of care and outcomes for an unselected cohort of patients across large health care treatment and referral networks or populations, as well as providing clinical decision-making guidance. Monitoring and reporting could be performed against agreed care standards and benchmarked outcomes for specific conditions, which would supplant the need for developing and maintaining labour intensive, condition-specific population-based observational cohort studies and clinical registries. A critical step of the methodology not fully described in this current paper is the requirement for validation studies comparing the clinically-defined EMR cohort definition against a gold standard to estimate sensitivity and specificity, prior to implementation into the production EMR. The gold standard depends on the context of how the EMR data will be used and could be clinician diagnosis if used for clinical decision support, or clinical registries or validation against ICD10 codes 
as previously performed in other conditions such as heart failure, post-traumatic stress disorder, Charleston Co-morbidity index, etc. [30, 38, 39]. A validation study estimating sensitivity and specificity for acute coronary syndrome comparing clinician diagnosis against ICD10 codes was performed in a 3 month EMR dataset from our current study; those results are currently under review [40].

The overarching strength of the research is the liberal nature of the data extraction process enabling future validation work critical for secondary use of EMR data, flexibility for creating new EMR-derived cohorts as clinical definitions and guidelines get updated as well as being able to identify and extract information on patients based on presenting symptoms and investigations, rather than diagnostic code. For example, using this generalisable methodology (Fig. 1) we can create EMR-derived cohorts for stroke, heart failure patients, etc. To our knowledge, this is also the first time that clinically-relevant information for diagnosing ACS has been collated from EMR data extracts (Additional file 1: Table S1). Limitations of the research include that the study period was restricted to extracted data from one information system (Cerner Millenium), chosen as it was the main EMR system, from only two of the fifteen local health districts in New South Wales and due to restricted access to the production EMR system, we were unable to iterate on the cohort identification process; this is possible when data extraction can occur on non-production EMR environments (i.e. clinical data warehouses) which are available at many sites in the UK and USA. Nevertheless the principles of this robust methodology can be applied to any EMR data extraction process and generalised to other diseases/ conditions. Extending data extraction processes across health jurisdictions and for other conditions will enable further validation of the methodology.

\section{Conclusion}

This paper demonstrates that clinically-defined EMR cohorts created using a broad strategy utilising structured and unstructured free-text in production EMR systems, are likely to identify relevant cohorts of patients and enable critical validation work required for real-time clinical decision support and secondary use of EMR data.

\section{Supplementary Information}

The online version contains supplementary material available at https://doi. org/10.1186/s12911-021-01441-w.

Additional file 1. List of search terms for Inclusion Criteria 1 \& 2, Approaches for ensuring data quality and study operational framework.

Additional file 2: Fig. S1. UpSet plot showing the number of eligible encounters meeting individual (bottom left hand side) and multiple inclusion criteria (right-hand side) in 2013-6. This UpSet plot represents eligible encounters from Cerner information systems in two local health districts that met at least one of the study inclusion criteria in $2013(n=135,511), 2014(n=143,108), 2015(n=163,008)$ and 2016 $(n=185,414)$. The bottom left-hand side represents the total number and percentage of encounters that met each inclusion criterion. Each inclusion criterion is represented as an independent group. The top right-hand side represents the number and percentage of encounters that met each combination of the inclusion criteria. Inclusion Criteria referred to: (1) Presenting problem key match: Keyword match in a free-text field for presenting information, (2) Triage information key match: The patient was assigned to a cardiac pathway mode of care or keyword match in the ED Triage descriptions, (3) Related Order: The existence of a cardiology-related order; (5) Related Procedure: the existence of cardiology-related procedure, (6) ICD-10/SNOMED CT: The encounter had an SNOMED CT or ICD-10 code for Acute Myocardial Infarction (AMI) and (7) Scanned ECG image: The encounter had a scanned ECG report available. Inclusion Criterion (4), representing patients that had a cardiac monitoring form, was excluded as no encounters met this inclusion criterion.

Additional file 3: Fig. S2. Correlation matrix examining associations between inclusion criteria.

\section{Abbreviations}

ACS: Acute coronary syndrome; CCL: Cerner Command Language; ECG: Elecrocardiogram; EMR: Electronic medical record; ICD-10: International Classification of Diseases and Related Health Problems (10th edition); LHD: Local Health District; NSW: New South Wales; SNOMED CT: SNOMED clinical terms; STEMI: ST-elevation myocardial infarction.

\section{Acknowledgements}

This project was supported by the Sydney Informatics Hub, funded by the University of Sydney. We would also like to thank Ms Seven Guney and Mr Matthew Strasiotto for their assistance on this project and A/Prof Adam Dunn for critical feedback on the manuscript.

\section{Authors' contributions}

Authors CST/DB wrote the first draft of the manuscript. Authors AS/RM performed data analysis. All authors (CST/JG/AS/SV/GF/CC/MC/RM/MW/JM/ DB) reviewed, provided critical feedback. All authors read and approved the final manuscript.

\section{Funding}

Funding for the SPEED-EXTRACT study was provided by grants from the Agency for Clinical Innovation, NSW Ministry of Health and Sydney Health Partners. Author CST was supported by a National Health and Medical Research Centre Early Career Fellowship from Australia (\#1037275). Author AS was supported by Sydney Health Partners ("Harnessing the EMR to improve care of patients with acute chest pain within Sydney Health").

\section{Availability of data and materials}

The datasets generated and/or analysed during the current study are not publicly available as they are owned by the Chief Executives of the Local Health Districts and not by the researchers who performed this study.

\section{Ethics approval and consent to participate}

Ethics and governance approval for the study (called SPEED-EXTRACT) was provided by the Northern Sydney Local Health District (NSLHD) Human Research Ethics Committee. We had a waiver of consent for the patients in this study which was approved by the NSLHD Human Resesarch Ethics Committee.

\section{Consent for publication}

Not applicable.

\section{Competing interests}

Not applicable. 


\section{Author details}

${ }^{1}$ Centre for Translational Data Science, The University of Sydney, Sydney, Australia. ${ }^{2}$ Northern Clinical School, The University of Sydney, Sydney, Australia. ${ }^{3}$ Susan Wakil School of Nursing and Midwifery, The University of Sydney, Sydney, Australia. ${ }^{4}$ Faculty of Health Sciences, The University of Sydney, Sydney, Australia. ${ }^{5}$ Cardiothoracic and Vascular Health, Kolling Institute of Medical Research and Department of Cardiology, Royal North Shore Hospital, Northern Sydney Local Health District, Sydney, Australia. ${ }^{6}$ Westmead Applied Research Centre, The University of Sydney, Sydney, Australia. ${ }^{7}$ Department of Cardiology, Westmead Hospital, Sydney, Australia. ${ }^{8}$ Clinical and Population Perinatal Health, Northern Sydney Local Health District, Sydney, Australia. ${ }^{9}$ Centre for Population Health, NSW Ministry of Health, Sydney, Australia. ${ }^{10}$ Department of Cardiology, Central Coast Local Health District and University of Newcastle, Sydney, Australia. ${ }^{11}$ Department of Obstetrics and Gynaecology, Northern Sydney Local Health District, Sydney, Australia. ${ }^{12}$ Department of Cardiology, Concord Hospital, Sydney, Australia. ${ }^{13}$ School of Computer Science (J12), The University of Sydney, Office 543, Level 5, Sydney, NSW 2006, Australia.

Received: 8 October 2020 Accepted: 15 February 2021 Published online: 08 March 2021

\section{References}

1. Casey JA, et al. Using electronic health records for population health research: a review of methods and applications. Annu Rev Public Health. 2016;37:61-81.

2. Haendel MA, Chute CG, Robinson PN. Classification, ontology, and precision medicine. N Engl J Med. 2018;379(15):1452-62.

3. Devine EB, et al. Automating electronic clinical data capture for quality improvement and research: the CERTAIN validation project of real world evidence. EGEMS (Wash DC). 2018;6(1):8.

4. De Coster $C$, et al. Identifying priorities in methodological research using ICD-9-CM and ICD-10 administrative data: report from an international consortium. BMC Health Serv Res. 2006;6:77.

5. Johnson EK, Nelson CP. Values and pitfalls of the use of administrative databases for outcomes assessment. J Urol. 2013;190(1):17-8.

6. Manuel DG, Rosella LC, Stukel TA. Importance of accurately identifying disease in studies using electronic health records. BMJ. 2010;341:c4226.

7. Burns EM, et al. Systematic review of discharge coding accuracy. J Public Health (Oxf). 2012;34(1):138-48.

8. Sutton RT, et al. An overview of clinical decision support systems: benefits, risks, and strategies for success. NPJ Digit Med. 2020;3:17.

9. Shivade C, et al. A review of approaches to identifying patient phenotype cohorts using electronic health records. J Am Med Inform Assoc. 2014;21(2):221-30.

10. Colborn $\mathrm{KL}$, et al. Identification of urinary tract infections using electronic health record data. Am J Infect Control. 2019;47(4):371-5.

11. Botsis TH, Chen F, Weng C. Secondary use of EHR: data quality issues and informatics opportunities. Summit on Translational Bioinformatics, 2010: p. $1-5$.

12. $\mathrm{Xu} \mathrm{H}$, et al. Extracting and integrating data from entire electronic health records for detecting colorectal cancer cases. AMIA Annu Symp Proc. 2011;2011:1564-72

13. McDonald $\mathrm{HI}$, et al. Methodological challenges when carrying out research on CKD and AKI using routine electronic health records. Kidney Int. 2016;90(5):943-9.

14. Al Sallakh MA, et al. Defining asthma and assessing asthma outcomes using electronic health record data: a systematic scoping review. Eur Respir J. 2017:49(6):1700204.

15. Ingram WM, et al. Defining major depressive disorder cohorts using the EHR: multiple phenotypes based on ICD-9 codes and medication orders. Neurol Psychiatry Brain Res. 2020;36:18-26.

16. Holve E, Segal C, Hamilton Lopez M. Opportunities and challenges for comparative effectiveness research (CER) with electronic clinical data: a perspective from the EDM forum. Med Care. 2012;50(Suppl):S11-8.

17. Sun $W$, et al. Data processing and text mining technologies on electronic medical records: a review. J Healthc Eng. 2018;2018:4302425.

18. Abhyankar $\mathrm{S}$, et al. Combining structured and unstructured data to identify a cohort of ICU patients who received dialysis. J Am Med Inform Assoc. 2014;21(5):801-7.
19. Carroll RJ, et al. Portability of an algorithm to identify rheumatoid arthritis in electronic health records. J Am Med Inform Assoc. 2012;19(e1):e162-9.

20. Kreuzthaler M, Schulz S, Berghold A. Secondary use of electronic health records for building cohort studies through top-down information extraction. J Biomed Inform. 2015;53:188-95.

21. Fernandez-Breis JT, et al. Leveraging electronic healthcare record standards and semantic web technologies for the identification of patient cohorts. J Am Med Inform Assoc. 2013;20(e2):e288-96.

22. Virani SS, et al. The use of structured data elements to identify ASCVD patients with statin-associated side effects: insights from the Department of Veterans Affairs. J Clin Lipidol. 2019;13(5):797-803e1.

23. Ford $E$, et al. Extracting information from the text of electronic medical records to improve case detection: a systematic review. J Am Med Inform Assoc. 2016;23(5):1007-15.

24. Healthstats, NSW. http://www.healthstats.nsw.gov.au/Indicator/dem_ pop_age/dem_pop_Ihn_snap 2020 1/2/20.

25. Benchimol El, et al. The REporting of studies Conducted using Observational Routinely-collected health Data (RECORD) statement. PLoS Med. 2015;12(10):e1001885.

26. Kirby JC, et al. PheKB: a catalog and workflow for creating electronic phenotype algorithms for transportability. J Am Med Inform Assoc. 2016;23(6):1046-52.

27. Conway JR, Lex A, Gehlenborg N. UpSetR: an R package for the visualization of intersecting sets and their properties. Bioinformatics. 2017;33(18):2938-40.

28. Pendergrass SA, Crawford DC. Using electronic health records to generate phenotypes for research. Curr Protoc Hum Genet. 2019;100(1):e80.

29. van Walraven C, Bennett C, Forster AJ. Administrative database research infrequently used validated diagnostic or procedural codes. J Clin Epidemiol. 2011;64(10):1054-9.

30. Kashyap $R$, et al. Derivation and validation of a computable phenotype for acute decompensated heart failure in hospitalized patients. BMC Med Inform Decis Mak. 2020;20(1):85.

31. Jackson RG, et al. Natural language processing to extract symptoms of severe mental illness from clinical text: the Clinical Record Interactive Search Comprehensive Data Extraction (CRIS-CODE) project. BMJ Open. 2017;7(1):e012012.

32. van Walraven C, Austin P. Administrative database research has unique characteristics that can risk biased results. J Clin Epidemiol. 2012;65(2):126-31.

33. Juhn Y, Liu H. Artificial intelligence approaches using natural language processing to advance EHR-based clinical research. J Allergy Clin Immunol. 2020;145(2):463-9.

34. Wu S, et al. Deep learning in clinical natural language processing: a methodical review. J Am Med Inform Assoc. 2020;27(3):457-70.

35. Review HB. Using Al to improve electronic medical records. 2018. https:// hbr.org/2018/12/using-ai-to-improve-electronic-health-records.

36. Penz JF, Wilcox AB, Hurdle JF. Automated identification of adverse events related to central venous catheters. J Biomed Inform. 2007;40(2):174-82.

37. Rasmussen LV, et al. Considerations for improving the portability of electronic health record-based phenotype algorithms. AMIA Annu Symp Proc. 2019;2019:755-64.

38. Harrington $\mathrm{KM}$, et al. Validation of an electronic medical record-based algorithm for identifying posttraumatic stress disorder in U.S. Veterans. J Trauma Stress. 2019;32(2):226-37.

39. Singh B, et al. Derivation and validation of automated electronic search strategies to extract Charlson comorbidities from electronic medical records. Mayo Clin Proc. 2012;87(9):817-24.

40. Saavedra A, Morris RW, Tam C, Killedar M, Ratwatte S, Huynh R, Yu C, Yuan DZ, Cretikos M, Gullick J, Vernon ST, Figtree GA, Morris J, Brieger D. Validation of acute myocardial infarction (AMI) in electronic medical records: the SPEED-EXTRACT study. 2020.https://www.medrxiv.org/conte nt/10.1101/2020.12.08.20245720v1.

\section{Publisher's Note}

Springer Nature remains neutral with regard to jurisdictional claims in published maps and institutional affiliations. 